\title{
A Coordenação Estratégica das Políticas de Colaboração Internacional em Ciência e Tecnologia
}

\author{
The Strategic Coordination of International Collaboration Science and Technology \\ Policy.
}

\section{Amanda Almeida Domingues ${ }^{1}$}

\section{RESUMO}

Este artigo tem por objetivo introduzir o debate a respeito da necessidade de delineamento de estratégias para as Políticas de Colaboração Internacional em Ciência e Tecnologia (C\&T). A principal hipótese demonstra que a formalização ou o delineamento de uma estratégia nacional ou multilateral para a colaboração internacional em C\&T depende do contexto político e científico no qual estão inseridos os atores responsáveis por estas e que outros fatores condicionantes devem ser investigados. A metodologia do trabalho está concentrada na análise de uma literatura pouco conhecida a respeito de estratégias de elaboração de políticas, bem como no estudo de documentos oficiais que congregam planos e políticas voltados ao estímulo da colaboração internacional em C\&T.

Palavras-Chave: Ciência e Tecnologia; Políticas Públicas; Colaboração Internacional.

\begin{abstract}
This article introduces the debate about the alleged necessity of strategic coordination of International Science and Technology (S\&T) Collaboration Policy. The arguments presented here were developed on grounds of the 2009 report of the European Union Commission which investigated S\&T international collaboration actors and strategies in 20 countries worldwide. The conclusion states that the formalization of a national or multilateral strategy for International Collaboration in S\&T depends on the political and scientific context in which the actors are inserted. In addition to it, other factors must be investigated.
\end{abstract}

Keywords: Science and Technology; Public Policy; International Collaboration.

\section{INTRODUÇÃO}

Este artigo trata de um tema atual e bastante discutido no país atualmente: a colaboração internacional ${ }^{2}$ em ciência e tecnologia (C\&T). Dentro desse grande âmbito

\footnotetext{
${ }^{1}$ Doutoranda em Ciência Política, Universidade de São Paulo, São Paulo, Brasil.

2 É importante citar que alguns teóricos, como Silva (2007), diferenciam os termos cooperação e colaboração. Segundo estes, colaboração é uma relação assimétrica e não equitativa, tendo, de um lado, um ator principal responsável pela parceria e proprietário dos resultados e, de outro, seus coadjuvantes. Já cooperação é uma relação mais igualitária que privilegia o diálogo, a negociação e a decisão conjunta, a definição de projetos em comum acordo e o compartilhamento de custos. Apesar de reconhecer que na prática há dois tipos de relações de parceria, uma mais assimétrica e uma mais equitativa, marcada mais pela coordenação do que pelo controle, no âmbito deste trabalho os dois termos serão tratados como semelhantes, preservando as diferenças existentes nas análises caso a caso.
} 
de pesquisa científica, este trabalho destaca o debate a respeito de estratégias para colaboração internacional em C\&T.

Alguns autores e políticos argumentam que a falta de uma coordenação estratégica das Políticas de Colaboração Internacional em C\&T seria a principal barreira para um aumento ainda mais expressivo das atividades colaborativas em C\&T e para uma maior eficácia destas no âmbito nacional e internacional. Ao longo do artigo, destaco os casos de Brasil, Estados Unidos e União Europeia, que revelam diferentes modelos para a cooperação internacional e para a articulação desta com a Política Científica e Tecnológica nacional. Analisando esses casos e considerando o relatório da Comissão Europeia para a cooperação internacional (2009), avanço uma resposta para a seguinte questão: seria mesmo necessário ter uma estratégia para a cooperação internacional para que os resultados destas parcerias fossem mais concretos e duradouros? 0 estudo constrói o argumento contra uma resposta positiva a essa questão.

\section{A COLABORAÇÃO INTERNACIONAL EM C\&T}

As colaborações internacionais em ciência e tecnologia (C\&T) têm apresentado inédito crescimento nas últimas décadas e a promoção de iniciativas para seu desenvolvimento passou a tomar a agenda de governos nos níveis nacionais e subnacionais, bem como de Instituições de Pesquisa e de Ensino Superior e agências de fomento - fatos que justificam seu estudo.

Colaborações Internacionais em C\&T consistem no trabalho conjunto de cientistas de dois ou mais países, cujo objetivo é concretizar determinados interesses, possibilitando o intercâmbio de conhecimento. Atualmente, grandes desenvolvimentos em C\&T - como os estudos sobre o Bóson de Higgs - são muitas vezes resultado de colaborações e/ou consórcios internacionais. A dimensão internacional é inerente à organização social da ciência (MERTON, 1977) e a produção do conhecimento se encontra, hoje, baseada cada vez mais na interação entre cientistas, indivíduos e organizações. A construção de bases de dados internacionais, organização de conferências, coleta e manutenção de fundos para um laboratório internacional, estabelecimento de padrões técnicos, assistência técnica e assistência ao 
desenvolvimento são somente alguns exemplos de cooperações internacionais em C\&T (WAGNER, 2005).

As atividades colaborativas podem se iniciar de modo formal, ou seja, sendo precedidas pela assinatura de um instrumento específico entre os parceiros, sejam eles indivíduos, países ou instituições. Nessa condição, elas representam formas estruturadas de cooperação com comprometimento a longo prazo, atividades concretas e contratos e seus atores operam o acordo de forma autônoma (BAUD, 2001). Além do modo formal, as colaborações podem se iniciar informalmente, não necessitando destes mecanismos. É bastante comum que colaborações internacionais se iniciem de modo informal e se formalizem com o tempo, envolvendo instituições e nações.

Quando a cooperação envolve órgãos do Estado, ela também representa um instrumento de Política Externa e de ampliação dos interesses externos de uma nação. Nesse sentido, a cooperação internacional em C\&T consiste em uma ação coordenada de dois ou mais Estados, com vistas a atingir resultados por eles julgados desejáveis e deve ser desenvolvida no conjunto do relacionamento internacional do país (AMORIM, 1994), pois ela é uma ferramenta poderosa de aprimoramento das relações internacionais, bem como de aproximação e manutenção de relações em outros campos, como o econômico, por exemplo.

Hoje, mais do que nunca, os Estados e outros órgãos nacionais e internacionais têm se preocupado com o estímulo às colaborações internacionais e têm buscado formular políticas que orientem as atividades colaborativas dos pesquisadores. As Políticas de Colaboração Internacional em C\&T consistem nessas ações explícitas dos governos oficiais (regulamentos, programas, acordos oficiais e memorandos de entendimento, investimentos financeiros, etc.) que têm por objetivo influenciar a intensidade, o conteúdo e a direção das colaborações entre pesquisadores através das fronteiras, sejam estes dos setores público ou privado (COMISSÃO EUROPEIA, 2009).

Por contemplar interesses científicos específicos, além de fazerem parte da Política Externa, as Políticas de Colaboração Internacional em C\&T são item da Política Científica e Tecnológica (PCT) dos Estados. Estes interesses científicos estão relacionados às vantagens proporcionadas pela colaboração como acesso a habilidades e conhecimentos complementares, aumento da visibilidade da pesquisa conduzida em parceria, aumento do impacto de produções científicas produzidas em parceria, 
compartilhamento de custos e equipamentos (WAGNER, 2000; GEORGHIOU, 1998; KATZ; MARTIN; 1997), entre outros.

As Políticas de Colaboração Internacional em C\&T também consistem em instrumentos relevantes para a realização de objetivos não relacionados à dinâmica científica, como o desenvolvimento econômico, o aprimoramento da competitividade nacional e o enfrentamento conjunto de desafios globais (mudanças climáticas, recursos energéticos renováveis), o investimento e o progresso da área da saúde e a construção de relações diplomáticas estáveis. Muitas aproximações políticas são iniciadas por acordos ou memorandos de cooperação em áreas científicas, pois a ciência é universalmente reconhecida e fomentada. Desta forma, as Políticas de Colaboração Internacional em C\&T são fundamentais, pois direcionam o desenvolvimento científico e tecnológico do país para aquelas áreas mais estratégicas e possibilitam seu gerenciamento.

\section{OS ATORES DA COLABORAÇÃO INTERNACIONAL EM C\&T}

No âmbito da colaboração informal, a unidade básica da cooperação em C\&T é o indivíduo pesquisador (KATZ; MARTIN, 1997) que representa a comunidade de pesquisa, o principal executor das atividades de colaboração internacional em C\&T. As comunidades científicas são os atores que possuem mais experiência com colaborações internacionais em C\&T e têm trabalhado em conjunto há séculos. Os pesquisadores são vistos pela sociedade como facilitadores que abrem canais de diálogo com outros atores, especialmente na indústria e no governo (SILVA, 2007). Os cientistas têm papel substancial tanto na execução da colaboração internacional em C\&T quanto na sua formulação, implementação e avaliação. A comunidade científica é a mola motriz dos projetos e programas de pesquisa desenvolvidos no âmbito internacional, o principal veículo de comunicação e integração com comunidades científicas de outros países e é também relevante ator da arena política - ao lado dos tomadores de decisões - nas discussões relacionadas à C\&T.

No entanto, quando se trata de colaborações formais, que exigem acordos institucionais, as colaborações internacionais podem se apresentar em diversos outros níveis, envolvendo diferentes atores. Elas podem ocorrer entre grupos de pesquisa, departamentos da mesma instituição, entre instituições e setores diferentes - 
universidades, empresas, centros de $\mathrm{P} \& \mathrm{D}$, laboratórios públicos e privados - entre regiões geográficas e países (KATZ; MARTIN, 1997).

Além destes níveis, no âmbito internacional, os atores científicos contam com a atuação de instituições supranacionais coordenadoras de projetos em cooperação com objetivos específicos, envolvendo diversos países. Exemplos exitosos de programas de cooperação internacional são o Programa Espacial Europeu, a Organização Europeia para Pesquisa Nuclear (CERN), o projeto Genoma e a Estação Espacial Internacional. Em relação a organizações científicas internacionais, é possível citar a Universal Society of Ophthalmology (primeira organização global de cientistas) e diversas outras (KERWIN, 1981).

As organizações supranacionais de pesquisa se apresentam de diferentes formas, sendo as instalações de pesquisa colaborativa como o CERN, as mais comuns. Há ainda instituições internacionais que conduzem pesquisa como o Instituto Europeu de Tecnologia, os programas internacionais em ciência, como o Human Frontiers Science Program e os financiadores internacionais de pesquisa como a Fundação Europeia para a Ciência. A maioria destas organizações é representada por colaborações formais entre governos e estabelecidas por tratados internacionais (COMISSÃO EUROPEIA, 2009).

Outros tipos de arranjos colaborativos são as chamadas sociedades profissionais e os acordos formais entre nações e grupos diversos financiados pelos governos, como por exemplo, a Organização do Tratado do Atlântico Norte (OTAN). Além de instituições supranacionais, atualmente a colaboração internacional em C\&T é também desenvolvida por atores subnacionais, como as universidades, organizações e institutos públicos de pesquisa, agências de fomento estaduais, entre outras.

Além dos atores já citados - governos nacionais, organizações de pesquisa multilaterais, organizações públicas de pesquisa, universidades e centros de pesquisa - é importante mencionar a forte atuação dos Ministérios diretamente responsáveis pela PCT e por outras áreas que utilizam as colaborações em C\&T como instrumentos para atingir suas missões políticas, como as áreas da agricultura, energia, meio ambiente, relações exteriores, assistência ao desenvolvimento e saúde. Além destes, diversas organizações não governamentais têm criado programas específicos para apoiar a pesquisa científica e um crescente número de embaixadas e representações estrangeiras passaram a oferecem inteligência estratégica em países específicos. 
Assim, vários são os atores responsáveis pela condução, manutenção e promoção de colaborações internacionais em C\&T e múltiplos são seus objetivos, metas, planos de ação e estratégias. 0 interesse no estudo destes novos atores advém do fato de legarem grande importância ao tema e investirem grandes quantias em projetos e programas colaborativos. Os atores estatais recebem, atualmente, grande destaque no âmbito da configuração das Políticas de Colaboração Internacional em C\&T. São notáveis as iniciativas de desenvolvimento e manutenção de colaborações em C\&T de Ministérios e departamentos responsáveis diretamente por questões de C\&T, agências e organismos financiadores e Ministérios responsáveis por outros temas, como saúde a agricultura.

Esta notoriedade advém do fato de que o Estado é um dos principais atores na formulação, execução e avaliação de políticas voltadas ao estímulo à colaboração internacional em C\&T. As atividades de C\&T são elementos essenciais para o desenvolvimento nacional, pois afetam, direta ou indiretamente, diversos aspectos da vida econômica e social. Da mesma forma, o Estado é vital para a concretização de atividades de C\&T, seja por meio de seu apoio político a acordos e parcerias, seja pelo comprometimento e investimento financeiro a projetos e programas. Desta forma, seu papel perpassa os dos demais atores, pois "somente um esforço coordenado a partir do Estado pode garantir o acesso ao conhecimento científico de fronteira internacionalmente disponível e sua adaptação e disseminação às realidades nacionais" (MCTI, 2002, p. 30).

O Estado participa do estímulo à colaboração internacional por meio de diversos órgãos e agências nacionais, regionais e até mesmo locais. Desta forma, ações de colaboração internacional são conduzidas por meio de uma série de estratégias de diferentes tipos de atores. Devido à multiplicidade de interesses em jogo, é possível afirmar que as Políticas de Colaboração Internacional em C\&T são menos um conjunto de medidas bem estruturado e mais uma coleção de medidas individuais tomadas por diferentes atores. A coordenação entre estes atores e políticas é uma questão de governança nacional e varia abruptamente de país para país.

Os Estados e a comunidade internacional reconhecem a importância estratégica da C\&T no mundo globalizado atual. Principalmente após o final da Guerra Fria, os Estados utilizam a colaboração internacional mais intensamente, combinando C\&T com economia e geopolítica (SILVA, 2007). 0 estímulo às atividades de colaboração concedido pelos governos, instituições, órgãos e agências de fomento, por meio de 
políticas públicas e estratégias de apoio, reflete o reconhecimento de que essas atividades avançam o conhecimento, trazem importantes benefícios, poupam custos e são um modo de aplicar mais efetivamente os resultados dos esforços científicos empreendidos (KATZ, 1994).

\section{AS ESTRATÉGIAS: DEFINIÇÃO E EXEMPLOS}

As Políticas de Colaboração Internacional em C\&T vem sendo cada vez mais utilizadas para atingir objetivos fundamentais dos Estados e instituições: promover o avanço da ciência e alcançar finalidades econômicas, políticas, diplomáticas. Porém, na consecução de políticas de estímulo à colaboração internacional, tanto o Estado quanto estes novos atores na esfera das colaborações internacionais em C\&T - agências de fomento, instituições de pesquisa, ministérios e outros - enfrentam desafios institucionais, políticos, jurídicos que comprometem a eficácia da formulação, execução e avaliação das Políticas de Colaboração Internacional em C\&T, apesar do forte estímulo que recebem.

0 estudo dos desafios enfrentados pelos atores envolvidos com atividades colaborativas é fundamental, pois a eficácia das Políticas de Colaboração Internacional em C\&T é comprometida pela não superação destas dificuldades, acarretando, assim, em ineficiência de programas e ações voltadas ao estímulo da colaboração e muitas vezes o gasto de recursos escassos em atividades que não trazem os benefícios esperados.

A problemática deste artigo jaz na concepção de alguns autores de que a falta de uma coordenação estratégica das Políticas de Colaboração Internacional em C\&T entre os atores envolvidos com a colaboração internacional é a principal barreira para um aumento ainda mais expressivo das atividades colaborativas em C\&T e para uma maior eficácia destas no âmbito nacional e internacional.

Uma estratégia política para a Colaboração Internacional pode ser definida como uma ideia unificadora e orientadora que organiza as Políticas de Colaboração Internacional em C\&T e que influencia e condiciona o processo político em torno de questões de colaboração internacional em C\&T, pois define o papel de cada ator no processo político e o que ele pode obter em cada circunstância (PAQUETTE, 2002). Esta definição não advém de teorias da administração ou de modelos de políticas públicas, 
mas ressalta justamente os aspectos que interessam a este trabalho: os aspectos operacionais de sua formulação.

As estratégias são compostas de quatro principais elementos: metas, táticas, ideia central (core idea) e estilo (PAQUETTE, 2002). As metas são os objetivos que se deseja alcançar com a implantação das ações e devem ser examinadas a partir de seu conteúdo. As táticas são os meios usados para alcançar os objetivos e estão essencialmente relacionadas às metas. A ideia central, chamada também de metáfora ou analogia, não é explícita, tendo que ser identificada por inferência. Ela direciona e coordena táticas e é expressa por um slogan ou um símbolo. A ideia central possibilita certa flexibilidade do estrategista, a tomada de decisões imediatas de membros do grupo sem a necessidade de uma consulta prévia ao primeiro e permite que o estrategista lide com incertezas em cenários alternativos, pois ela reflete o conteúdo específico das ações a serem empreendidas. Por fim, o estilo da estratégia é a abordagem, os princípios que orientam a implantação desta. Eles são ideias gerais (que guiam a seleção de táticas) compartilhadas pelos membros do grupo que empreende a estratégia e que o diferencia de outros grupos (PAQUETTE, 2002).

Os valores dos atores envolvidos na formulação de estratégias consistem em um dos principais aspectos na determinação dos componentes da estratégia explicados acima (as metas, táticas, ideia central e estilo) (PAQUETTE, 2002). Valores podem ser definidos como heranças e padrões característicos de um indivíduo ou grupo que mudam em um ritmo muito devagar. A herança consiste na parte dos valores que pode ser passada de geração para geração e seus principais componentes intangíveis são as crenças - "afirmações existenciais consideradas como fatos por impulsos biológicos ou sociais" (PAQUETTE, 2002, p. 10, tradução nossa) - e os conhecimentos. (PAQUETTE, 2002).

Valores são compartilhados por um grupo que, em muitos casos, é caracterizado por uma combinação única e específica de certos valores. Os membros de uma instituição ou de certa comunidade (como a comunidade de pesquisa) compartilham valores por pertencerem a um mesmo grupo com características específicas e refletem estes valores na tomada de decisão.

As estratégias de Colaboração Internacional em C\&T são delineadas pelos atores que participam das colaborações internacionais, que devem definir objetivos, metas 
delimitadas e táticas. A estratégia está intimamente relacionada com os valores do indivíduo ou da instituição que a concebe.

É importante ressaltar que a estratégia à qual me refiro neste trabalho é a estratégia formal, ou seja, aquela que representa os procedimentos oficiais dos atores responsáveis pelas colaborações internacionais expressos em leis, regulamentos e estatutos (HERRERA, 1975) e que estão, em geral, divulgadas publicamente. Existem também as estratégias informais (expressão das expectativas e das demandas dos atores) que não são divulgadas e, por não serem facilmente identificáveis, necessitam de um estudo aprofundado. A adoção de uma estratégia formal não exclui a implementação concomitante de uma estratégia informal: muitas instituições proferem discursos em certa direção, mas implementam ações em outra.

A União Europeia talvez seja o melhor exemplo de instituição atuante na área de C\&T que busca articular seus membros em uma estratégia comum de colaboração internacional. Um a cada cinco projetos de pesquisa na União Europeia conta com a participação de parceiros internacionais (ISC, 2013). Por isso, a instituição criou, em 2008, o Strategic Framework for International Science and Technology Cooperation (COMISSÃO EUROPEIA, 2008) cujo objetivo é estabelecer princípios gerais e ações que eliminem o investimento duplicado, inaugurem prioridades para a C\&T e assegurem um esforço conjunto coerente dos Estados-membros.

Os Estados Unidos aprovaram recentemente (Junho de 2012) uma lei que vinha sendo negociada desde 2009 para a coordenação das atividades colaborativas do país. 0 documento cria um órgão específico sob os auspícios do National Science and Technology Council (NSTC) que tem como responsabilidades principais planejar e coordenar as atividades e parcerias para colaboração internacional em C\&T e estabelecer prioridades federais para as mesmas (ESTADOS UNIDOS DA AMÉRICA, 2012).

No Brasil, os principais financiadores da colaboração internacional são o Governo Federal - por meio do Conselho Nacional de Desenvolvimento Científico e Tecnológico (CNPq), da Coordenação de Aperfeiçoamento de Pessoal (CAPES), da Agência Brasileira de Cooperação (ABC) e da Financiadora de Estudos e Projetos (FINEP) - e as agências estaduais de fomento, com destaque para a Fundação de Amparo à Pesquisa do Estado de São Paulo (FAPESP).

O Ministério de Ciência, Tecnologia e Inovação (MCTI) lançou, em 2011, a Estratégia Nacional de Ciência, Tecnologia e Inovação 2012-2015. Este é o mais recente 
documento que contém diretrizes de ação para as atividades gerais de Ciência, Tecnologia e Inovação (CTI) dos órgãos sob responsabilidade do ministério. 0 documento também aborda direcionamentos para atividades de colaboração internacional. No entanto, esta estratégia se aplica somente àquelas instituições sob o comando do Ministério, excluindo importantes agentes da colaboração internacional, como a ABC, a CAPES e as agências estaduais. CAPES, FINEP e FAPESP não possuem estratégias formalizadas para a colaboração internacional em C\&T.

\section{AS ESTRATÉGIAS: A COLABORAÇÃO INTERNACIONAL EM C\&T E A POLÍTICA CIENTÍFICA E TECNOLÓGICA}

A Política de Colaboração Internacional em C\&T é um item importante da Política Científica e Tecnológica (PCT). Portanto, esperar-se-ia que a primeira estivesse articulada com a segunda e que ambas construíssem estratégias e metas de forma conjunta e que se apoiassem mutuamente. Porém, não é isso que acontece em todas as situações.

A maioria dos países possuem planos de metas ou estratégias gerais de PCT que delineiam o status quo e os objetivos futuros para a PCT como um todo. Não é raro encontrar, nestes planos e relatórios, referências à colaboração internacional. No entanto, alguns países e blocos regionais, como a União Europeia (UE), possuem documentos como este, porém específicos para a cooperação internacional. Portanto, há duas situações: países que possuem estratégias para a PCT e para a cooperação internacionais em documentos separados, e países que agregam estratégias para ambas em um só documento.

No entanto, é preciso notar que estando previstas em um plano específico ou contidas nas metas gerais para a PCT, as estratégias para a cooperação internacional não estão necessariamente articuladas à PCT geral. No caso da UE há uma clara correspondência entre a PCT do bloco e seus relatórios estratégicos para a cooperação internacional. As metas e objetivos delineados em ambos são correspondentes, articulados e dependentes. Porém, há casos como o do Brasil, onde não há estratégias específicas para a colaboração internacional (estas estão contidas na estratégia geral de PCT), e suas metas e objetivos parecem desvinculados e fora da estratégia geral de PCT. 
Nos últimos relatórios do MCTI a respeito da PCT brasileira, pouco é citado especificamente sobre cooperação internacional. Além disso, há diversas outras agências que conduzem atividades significativas de cooperação internacional que não seguem (e nem deveriam?) as estratégias delineadas pelo MCTI. Na verdade, um documento estratégico sobre a PCT brasileira deveria englobar metas e objetivos do MCTI, MRE e do Ministério da Educação (MEC), mas o que existe é uma total desarticulação e desconhecimento das atividades feitas por estes ministérios no campo da cooperação internacional.

Estes fatos a respeito do Brasil e da UE não comprovam que ter uma estratégia desvinculada da PCT, como o caso da UE, garante a correspondência entre PCT e a Políticas de Colaboração Internacional em C\&T. Os Estados Unidos também não possuem uma estratégia específica para colaboração internacional, mas sua articulação com a PCT é mais forte do que no caso brasileiro. Talvez seja o caso de se analisar o exemplo brasileiro.

No Brasil, a falta de coordenação entre a PCT e as Políticas de Colaboração Internacional advém do fato de que a própria PCT brasileira é desarticulada. Esse é um problema causado por deficiências de gestão e desarticulações institucionais. Muitos teóricos defendem que a PCT, por lidar com a autonomia dos pesquisadores e cientistas, seria desarticulada "por natureza", ou seja, pela necessidade de defender certa autonomia para os pesquisadores e ao mesmo tempo lidar com as demandas da sociedade. Porém, isso não é verdade. No Brasil, temos exemplos de gestões bemsucedidas, como a da Fundação de Amparo à Pesquisa do Estado de São Paulo, por exemplo, na qual a própria burocracia da instituição vem da comunidade científica e, portanto, compreende os desafios de agente e de principal, ou seja: os desafios do agente que conduz a pesquisa e necessita de autonomia para suas pesquisas, e os do principal que precisa de respostas à sociedade, financiando aquilo que responderá a demandas desta.

O erro do Governo Federal talvez esteja em construir uma burocracia (o MCTI e o MRE) que desconhece o cotidiano científico e que, portanto, não divide com os cientistas suas dificuldades e aspirações. Se afirmarmos que a falta de coordenação da PCT brasileira está relacionada à garantia de certa autonomia ao cientista, estamos dando passos em direção àqueles que acreditam que a ciência pode, de algum forma, ser neutra. 
A discussão a que este artigo se dedica, a da necessidade de se ter uma estratégia para cooperação internacional, tem como uma de suas consequências o afastamento da ideia de que os cientistas devem ser somente cientistas e zelar por sua autonomia e os burocratas devem ser somente burocratas e cumprir metas da administração. Talvez os modelos bem sucedidos de estratégias para a PCT e para a cooperação internacional assim o são porque quebraram esta fronteira e colocaram, na administração, pessoas que façam ambos os papeis - burocracia e ciência. No entanto, afirmo, novamente, que isso não quer dizer que ter uma estratégia para cooperação internacional em C\&T é essencial e algo que deve ser buscado.

\section{O DESAFIO DA CONCEPÇÃO DE ESTRATÉGIAS}

Para os órgãos dos Estados, um dos principais desafios atuais em relação à elaboração de estratégias de colaboração internacional é a estruturação desta de forma sólida e coordenada. Segundo o relatório da Comissão Europeia (COMISSÃO EUROPEIA, 2009) que analisou a colaboração internacional de 20 países europeus e de outros continentes, nenhum país examinado possui uma estratégia coerente e coordenada para a colaboração internacional em C\&T e têm atores organizados de forma explícita.

Os obstáculos para a concepção de uma estratégia advêm, principalmente, da operacionalização da cooperação internacional, ou seja, da própria organização, negociação e definição das características das parcerias. A direção geográfica da colaboração é feita de forma ad hoc, ou seja, é definida caso a caso e se pauta pelos benefícios científicos, políticos e econômicos esperados. As prioridades temáticas são estabelecidas de forma implícita ou ex post, ou seja, após a definição dos parceiros e do modo como a atividade será conduzida. Em raros casos há diretrizes para disciplinas científicas específicas: em geral, a colaboração está baseada em objetivos amplos e globais (COMISSÃO EUROPEIA, 2009). Além disso, por falta de conjugação de esforços dos atores envolvidos, não há coordenação, reciprocidade e acompanhamento adequado dos programas, que acabam propondo e realizando objetivos diversos.

Um dos mais importantes desafios enfrentados pelos Estados na construção de estratégias nacionais de colaboração internacional em C\&T é o ajuste dos compromissos assumidos com parceiros às legislações nacionais, - o que, muitas vezes, exige longos 
acordos diplomáticos, com até mesmo eventuais ajustes no sistema nacional de C\&T (COSTA FILHO, 2006).

A formulação de uma estratégia entre os parceiros também encontra obstáculos advindos de diferenças culturais entre estes, de nível de conhecimento, diferentes interesses científicos e de formas de financiamento à C\&T (devido ao tamanho do país ou ao diferente apoio às várias estruturas políticas), além de barreiras psicológicas, legais, políticas e burocráticas ${ }^{3}$. Um exemplo interessante é o fato de que algumas instituições ou países estão acostumados a lidar com a colaboração internacional de forma top-down, ou seja, as decisões a respeito de parcerias em C\&T advêm de instâncias superiores dos governos. Outros preferem a forma bottom-up na qual os pesquisadores têm liberdade de fazer propostas de projetos e parcerias e são consultados por órgãos responsáveis por estas. Abordagens que enfatizam direcionamentos exclusivamente topdown podem acarretar em perda de liberdade dos cientistas que acabam perdendo também o interesse em participar das colaborações. As abordagens bottom-up concedem grande autonomia aos cientistas, mas por outro lado, não dão o foco necessário aos recursos que são sempre limitados.

Em relação a agências, órgãos subnacionais e outras instituições, algumas ainda enfrentam dificuldades nas primeiras etapas da colaboração internacional: elas lutam com o design e a implementação de estratégias, bem como com a determinação do nível e tipo de envolvimento internacional que desejam (COMISSÃO EUROPEIA, 2009).

\section{TER OU NÃO TER?}

A atuação internacional, apesar de ser uma preocupação política há muitos anos, somente recentemente ganhou a atenção dos legisladores como uma dimensão política de C\&T que engloba diversas atividades e pode ser explorada de forma mais estratégica. Por isso, os atores envolvidos com decisões a respeito de colaborações internacionais

\footnotetext{
3 Há também a barreira da língua, visto que os mais renomados periódicos são publicados em língua inglesa. Para mais detalhes: Schwartzman, Simon. Catching up in science and technology: self-reliance or internationalization? Paper apresentado na seção "Nationalism and Internationalism of Science", no comitê de pesquisa em sociologia da ciência e da tecnologia. International Sociological Association World Congress, Beielefeld, Germany, July 18-23, 1994. Dwyer, Tom. Notes on some difficulties relating to the internationalization of Brazilian academic sociology. Paper apresentado na Conference of the Council of National Associations of the International Sociological Association. Academia Sinica, Taipei, 23-25 March 2009.
} 
em C\&T estão no início de um processo longo de aprendizado que certamente alcançará o desenvolvimento de políticas melhor estruturadas e objetivas.

Alguns entre eles acreditam que este desenvolvimento pode ser alcançado mais rapidamente por meio da estruturação de estratégias voltadas à coordenação das diversas Políticas de Colaboração Internacional em C\&T: sejam elas de órgãos ou instituições do mesmo Estado ou políticas que regem acordos multilaterais ou bilaterais. Como cada ator envolvido nas atividades colaborativas possui lógica e funcionamento próprios, as Políticas de Colaboração Internacional em C\&T executadas pelos vários atores podem acabar por apresentar discrepâncias e, certas vezes, duplicação de esforços em um determinado tema.

Estratégias bem estruturadas permitem que ideias complexas sejam compreendidas pelos atores envolvidos no ciclo das políticas, pois é muito comum que os estrategistas responsáveis pelo desenho das estratégias não sejam os executores das políticas. Por isso, a estratégia serve como guia para orientar esses últimos, que muitas vezes necessitam realizar adaptações e precisam de direcionamentos de como fazê-las. Além disso, por exigir uma delimitação grande de objetivos e ações, a estratégia aproxima os atores de resultados positivos, pois permite a concentração de esforços em ações consideradas mais adequadas. Além disso, uma ação coordenada evita a duplicação de esforços e gastos de recursos escassos em atividades que não trazem os benefícios esperados ou que não proporcionam o avanço social e econômico do país.

As características da maior parte das colaborações internacionais atuais já citadas (a falta de conjugação de esforços, a definição ex post das prioridades temáticas, a direção geográfica $a d$ hoc e etc.) prejudicam e até mesmo impedem a coordenação entre os atores envolvidos, o que compromete a eficácia das políticas produzidas em parceria. A distância entre os parceiros gera uma falta de conhecimento mútuo que se reflete na dificuldade de planejar próximos passos, pois não é possível prever as ações do parceiro e nem mesmo identificar com precisão seus objetivos.

Apesar destes argumentos, alguns atores envolvidos nas colaborações internacionais revelam que não há evidências de que a adoção de estratégias formais leve necessariamente a uma forte internacionalização ou a atividades colaborativas mais eficientes (COMISSÃO EUROPEIA, 2009). Pelo contrário, dos países analisados pelo relatório da Comissão Europeia, nenhum possui uma articulação forte. Nessa lista estão incluídos os Estados Unidos e a Inglaterra. 
No estudo de estratégias de colaboração internacional em C\&T é preciso diferenciar as estratégias formais das informais. Alguns atores trabalham com estratégias formais; outros só praticam estratégias informais. Há ainda um terceiro caso, no qual o ator possui ambas e estas não são congruentes, ou seja, há uma estratégia formal divulgada, porém as ações concretas são orientadas por outra estratégia, informal.

A adoção de uma estratégia informal não impacta na eficiência das políticas de colaboração internacional em C\&T, pois ela também é capaz de coordenar atores e gerir a colaboração internacional de forma adequada. Como ensina Dye (1992), a inação, ou seja, o fato de um ator não possuir uma estratégia formal, pode ser também uma estratégia. Nestes casos, é preciso entender porque alguns países ou instituições não possuem estratégias formais ou não as divulgam.

\section{CONSIDERAÇÕES FINAIS}

Os atores responsáveis pelas Políticas de Colaboração Internacional em C\&T atualmente enfrentam diversos desafios que comprometem a definição e coordenação estratégica destas políticas. Muitos teóricos do tema refletem a respeito da necessidade de criação de uma estratégia nacional ou até mesmo multilateral para coordenar as Políticas de Cooperação Internacional em C\&T.

Por um lado, a carência de estratégias bem definidas pode representar uma importante barreira ao crescimento ainda mais expressivo das parcerias internacionais em C\&T e também à obtenção de resultados mais efetivos. Por outro, a percepção de que a carência de estratégias políticas pode representar uma barreira a este crescimento pode estar equivocada, pois o fato de os atores não possuírem uma estratégia formal para a colaboração internacional, não leva necessariamente a políticas sem orientação e direcionamento ou vazias e sem coerência: diversos exemplos internacionais provam este argumento.

O fato é que a colaboração internacional em C\&T possui atualmente significativa importância para o desenvolvimento social e econômico dos Estados Nacionais, pois proporciona benefícios não somente no campo da C\&T, mas também em outros campos, como o econômico, social e político. Desta forma, estudar as Políticas de Colaboração Internacional em C\&T, em especial da perspectiva dos Estados - atores fundamentais 
devido ao seu papel de formulador de políticas e financiador de pesquisas - é essencial, pois seu estudo possibilita a condução de avaliações de eficácia e impacto, que somente podem ser realizadas se os objetivos e as formas como estas foram conduzidas estiverem claros para o investigador.

Este trabalho não pretende ser normativo. Não há uma resposta exata à pergunta que me propus a responder: ter ou não ter estratégia para colaboração internacional em C\&T? Cada país ou instituição opta por determinados mecanismos de coordenação de políticas (sejam eles estratégias formais, informais ou outro tipo de mecanismo) que sejam adaptados a seu contexto e objetivos específicos, ou seja, eles provavelmente são díspares não somente entre Estados diferentes, mas também entre instituições que lidam com temas de C\&T de um mesmo Estado. Alguns dentre estes têm sucesso em coordenar políticas e aprimorar a eficiência destas; outros permanecem no mesmo estágio que se encontravam anteriormente a elas. Um segundo grupo de países e/ou instituições opta por não adotar nenhum destes mecanismos, mas mesmo assim acabam experimentando uma forte internacionalização da C\&T e colaborações eficientes. E ainda há o caso de países ou instituições que também não se articulam, mas sofrem graves deficiências em seu sistema colaborativo. Portanto, a opção por uma determinada estratégia ou pela inação não leva necessariamente a uma melhor coordenação e eficiência de políticas.

Por estarem inseridos em sistemas nacionais diversos, lógicas econômicas e científicas diferentes e culturas e contextos variados, são os atores nacionais os mais bem preparados para avaliar a necessidade de estratégias ou planos de articulação política. Se o contexto é marcado por uma constante coordenação entre os atores, como é o caso da União Europeia, é provável que os países prefiram uma articulação. Porém, se o contexto é de atuação individual, como é o caso do Brasil - onde os ministérios são pouco articulados em qualquer matéria e no caso do financiamento da colaboração internacional, muitas das agências relevantes são independentes do MCTI (FAPESP, CAPES, universidades) - uma coordenação pode não ser adequada ou desejada.

Desta forma, a hipótese de que não é necessário ter uma estratégia para a cooperação internacional para que os resultados destas parcerias fossem mais concretos e duradouros se confirma. O argumento que é reforçado neste artigo é que a formalização ou o delineamento de uma estratégia nacional ou multilateral para a colaboração internacional em C\&T depende do contexto político e científico no qual 
estão inseridos os atores responsáveis por essas. Não é possível estabelecer uma normatividade para a necessidade de estratégias para as colaborações internacionais em C\&T, pois é possível observar casos eficientes tanto em países e instituições com estratégias bem estruturadas como naqueles que não possuem estratégia alguma. Há diversos outros fatores que influenciam a eficiência das Políticas de Colaboração Internacional em C\&T, bem como o crescimento das parcerias internacionais e que merecem estudo e atenção.

\section{REFERÊNCIAS}

AMORIM, C. L. N. 1994. Perspectivas da Cooperação Internacional. IN: MARCOVITCH, Jacques. Cooperação Internacional: Estratégia e Gestão. São Paulo: EDUSP.

BAUD, I. 2001. "North-South Partnerships in Development Research: An Institutions Approach". Royal Netherlands Academy of Arts and Sciences International Conference, Amsterdam, vol. 1, Dezembro.

COMISSÃO EUROPÉIA. 2009. Drivers of International collaboration in research. Bruxelas: Tecnopolis Group.

COMISSÃO EUROPEIA. 2008. European partnership for international scientific and technological cooperation. [Online]. Comissão Europeia. Disponível em: < http://register.consilium.europa.eu/pdf/en/08/st16/st16763.en08.pdf > . Acesso em: $17 / 05 / 2013$.

COSTA FILHO, Eduardo. 2006. A dinâmica da cooperação espacial sul-sul: o caso do programa CBERS. Campinas. Tese de Doutorado, Universidade Estadual de Campinas. Campinas.

DYE, Thomas. 1992. Understanding public policy. Londres: Prentice-Hall.

ESTADOS UNIDOS DA AMÉRICA. 2012. International Science and Technology Cooperation Act of 2012. [Online]. GovTrack.us. Disponível em: < https://www.govtrack.us/congress/bills/112/hr5916/text>. Acesso em: 22/08/2013.

GEORGHIOU, Luke. 1998. "Global Cooperation in Research". Research Policy, v. 27, n. 6, p. 611-626. 
HERRERA, Almicar. 1975. "Los Determinantes Sociales de la Política Científica en América Latina: Política Científica Explícita y Política Científica Implícita". Desarollo Econômico, Buenos Aires, vol. 13, n.49, 1975.

ISC. 2013. International Cooperation: Intelligence in Science. Disponível em: < http://www.iscintelligence.com/tema.php?id=6 >. Acesso em: 17/05/2013.

KATZ, J. 1994. Geographical proximity and scientific collaboration. Scientometrics, Amsterdam, v. 31, n. 1, p. 31-43.

KATZ; MARTIN. 1997. “What is Research Collaboration?” Research Policy, vol. 26, p. 118.

KERWIN, L. International Science: an overview. Science, v. 213, set., 1981.

MINISTÉRIO DA CIÊNCIA, TECNOLOGIA E INOVAÇÃO (MCTI). 2002. Livro Branco: Ciência, Tecnologia e Inovação. Brasília: Ministério de Ciência, Tecnologia e Inovação.

MERTON, Robert. 1977. La sociologia de la Ciencia. Madrid: Alianza Editorial SA.

PAQUETTE, Laure. 2002. Political Strategy and Tactics: a practical guide. Nova York: Nova Science Publishers.

SILVA, D. 2007. “Cooperação internacional em ciência e tecnologia: oportunidades e riscos.” Revista Brasileira de Política Internacional, Brasília, vol. 50, n 1.

WAGNER, Caroline. 2000. International Cooperation in research and development: an update to an inventory of U.S. government spending. Santa Monica: RAND.

WAGNER, Caroline 2005. "Six cases studies of international collaboration in science." Scientometrics, vol. 62, no 1 , p. 3-26. 